\title{
BINARITY OF THE NEARBY 30 Myr OLD SOLAR ANALOG HIP 16853 IN THE TUCANA-HOROLOGIUM STREAM
}

\author{
VALERI V. MaKarov \\ Michelson Science Center, California Technology Institute, Pasadena, CA; vvm@caltech.edu \\ Received 2006 October 24; accepted 2006 November 16; published 2006 December 18
}

\begin{abstract}
A robust unconstrained orbital solution is obtained for the G2 V star HIP $16853=$ HD 22705 at 42 pc, which is a probable member of the $28-30$ Myr old Tucana-Horologium stream of post-T Tauri stars. The solution yields an apparent semimajor axis of $5.1 \pm 0.7$ mas, a period of $201 \pm 2$ days, and an inclination of $80^{\circ} \pm 7^{\circ}$. Assuming a mass of $1 M_{\odot}$ for the primary, the close companion is only $0.4 M_{\odot}$, which implies a spectral type M0.5 at this age. The expected maximum separation ( $a$ ) between the companions is 18 mas, which makes this system amenable for high-resolution observations. The wide companion HIP 16853 B at 14" is investigated as a possible tertiary component but rejected on account of the near-infrared photometric data inconsistent with the well-defined $\mathrm{H}-\mathrm{R}$ diagram of the Tucana-Horologium group.
\end{abstract}

Subject headings: astrometry — binaries: close -

open clusters and associations: individual (Tucana-Horologium) stars: individual (HIP 16853)

The star HIP 16853 (HD 22705) is a very young analog of the Sun at a distance of $42 \mathrm{pc}$ from the Earth. It is a probable member of the stream of post-T Tauri stars found in the constellations of Tucana and Horologium in the southern sky (Torres et al. 2000; Zuckerman \& Webb 2000). Owing to the proximity of this group and a relatively high proper motion on the

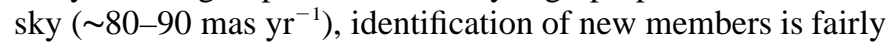
easy. HIP 16853 is a recent addition to the list of proposed members (Zuckerman \& Song 2004). The youth of this star is also betrayed by the remarkable chromospheric activity reported in Henry et al. (1996) with a Ca II H and $\mathrm{K}$ lines emission of $\log R^{\prime}(\mathrm{HK})=-4.33$. The star passes some of the selection criteria for TUCHOR membership in Makarov (2007), including a few close conjunctions with other members in the past; however, no close encounter with the Upper Scorpius OB association (or its progenitor cloud) has been found, which is considered to be the likely origin of TUCHOR, or of its major part. A possible reason for this miss is that the radial velocity used in Makarov (2007) is perturbed by the orbital motion of the bright primary star around the center of mass in this fairly close binary. Indeed, HIP 16853 was found to be a single-lined spectroscopic binary in Nordström et al. (2004). A radial velocity of +14.4 is given in this extensive spectroscopic survey with a formal error of $0.9 \mathrm{~km} \mathrm{~s}^{-1}$ and a rms scatter of $4 \mathrm{~km} \mathrm{~s}^{-1}$ on 18 measurements. The mean radial velocity may still be affected by the orbital variation, since no orbital solution has been published.

HIP 16853 is also an astrometric binary with accelerating proper motion (ESA 1997; Makarov \& Kaplan 2005). An ac-

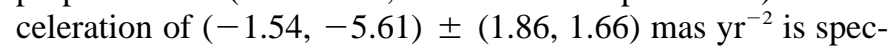
ified in the Hipparcos catalog. Despite its statistically significant value in declination, this determination is spurious, because the model of accelerated motion is not applicable in this case. Such spurious acceleration determinations occur when a relatively short period system $(P<3 \mathrm{yr})$ is sampled nonuniformly along its orbit according to the scanning law of Hipparcos. Meaningful parameters can only be derived from complete astrometric orbital solutions.

In the absence of a spectroscopic orbital solution, I compute an unconstrained 12-parameter fit on the 78 individual data points available in the Hipparcos Intermediate Astrometry Data (HIAD; ESA 1997). More accurate fits can be obtained in constrained astrometric solutions when spectroscopic estimates of $e, P, T$, and $\omega$ are used as constraints (e.g., Torres et al. 2003; Jancart et al. 2005). The technique of unconstrained orbital solutions and the difficulties arising in it are discussed in detail in Goldin \& Makarov (2006). For this star, however, there was no need in running the computationally heavy genetic optimization program described in that paper, because the orbital signature was so clear and prominent that a sufficiently good initial approximation could be done by eye. After a few iterations using the regular Powell optimization algorithm, the fit converged to the set of parameters specified in Table 1 . The formal reduced $\chi^{2}$ drops from 1.56 to 0.93 on 78-12 degrees of freedom. It should be mentioned that the formal $F$-test (which yields a confidence of 0.9997 for this orbital fit) may be significantly hampered by the strong nonlinearity and intrinsic correlations of the parameters $e, P$, and $T$, and the computed confidence is likely to be strongly overestimated.

The orbit of the primary component has a semimajor axis of about 5 mas and is inclined at a large angle (almost edgeon) to the plane of the sky. With a period of roughly 200 days, assuming a mass of $1 M_{\odot}$ for the primary component (spectral type G2), the total semimajor axis is $a=0.75 \mathrm{AU}$, or 18 mas on the sky, and the secondary mass is $0.4 M_{\odot}$. At the maximum separation, the companion can be resolved with high-resolution observations in the near-infrared, for example, by the new technique of spectroastrometry in combination with adaptive optics. Note that the parallax has not changed in the orbital solution with respect to the value specified in the Hipparcos catalog. The fit is consistent with $e=0$ (circular orbit). For this reason, the parameters $T$ (periastron time) and $\omega$ (longitude of periastron) become degenerate in the multiparameter least-squares adjustment and in the covariance calculus described in Goldin \& Makarov (2006).

A Hertzsprung-Russell (H-R) diagram based on the $J$ and $K_{s}$ magnitudes from the Two Micron All Sky Survey (2MASS) survey and the Hipparcos parallaxes is shown in Figure 1 for the following members of the Tucana-Horologium group: HIP 1113, 1481, 9141, 9892, 9902, 16853, 30034, 32435, 107947, and 108195. The star under consideration, HIP 16853, is marked with a cross on the graph. For comparison, two model isochrones for age $27 \mathrm{Myr}$ and $Z=0.01$ are shown from Siess 
TABLE 1

Orbital Parameters of HIP 16853

\begin{tabular}{lc}
\hline \hline \multicolumn{1}{c}{ Parameter } & Value \\
\hline$a_{0}$ (mas) $\ldots$ & $5.1 \pm 0.7$ \\
$P($ days $) \ldots$ & $201 \pm 2$ \\
$i($ deg $) \ldots$ & $80 \pm 7$ \\
$e \ldots$ & $0.0 \pm 0.3$ \\
$\Omega(\operatorname{deg}) \ldots$ & $36 \pm 7$ \\
Parallax (mas) $\ldots$ & $24.0 \pm 0.5$ \\
$\omega($ deg $) \ldots$ & $\simeq 65$ \\
$T_{0} \ldots$ & $\simeq \mathrm{JD} 2,448,400$ \\
\hline
\end{tabular}

et al. (2000, solid line) and Pietrinferni et al. (2004, dashed line). An age of $27 \mathrm{Myr}$ was inferred from kinematical considerations in Makarov (2007), where a past encounter of TUCHOR with the Upper Scorpius OB association was detected. Generally, the stars seem to follow the Siess et al. (2000) isochrone very well, except for the star HIP 108195, which has a $K$-band excess of over 1 mag. In particular, the star HIP 16853 lies very close to the Siess et al. isochrone. It may even be shifted by the expected 0.1 mag upward (to brighter $K_{s}$ ) by the contribution of light from the astrometric companion. However, this agreement cannot be considered a significant proof that the group as a whole and our object are indeed $27 \mathrm{Myr}$ old. Inconsistencies between the theoretical models are obvious; moreover, solar-like stars do not provide accurate age determination because the isochrones are already fairly compressed at this age. For example, a $1 M_{\odot}$ star at 27 Myr will be brighter than a similar star on the zero-age main sequence (ZAMS) by only $0.2 \mathrm{mag}$ in $J$ and in $K_{s}$, an amount within the model uncertainty. This difficulty motivates many researches to look for late-type members of young $\mathrm{T}$ associations, where the upturn of the main sequence becomes large. A $0.4 M_{\odot}$ star, by contrast, will have a magnitude difference of 0.66 between $27 \mathrm{Myr}$ and ZAMS in both $J$ and $K_{s}$.

We therefore should examine the faint visual companion HIP 16853 B, listed in the CCDM (Components of Double and Multiple Stars) catalog of double stars (Dommanget \& Nys 1994). It is located at $14.6^{\prime \prime}$ from the primary star at position angle $259^{\circ}$, according to CCDM. Hipparcos apparently did not resolve the alleged $\mathrm{B}$ companion, probably because of its optical faintness $(11.8 \mathrm{mag})$. This star is, however, listed in the 2MASS catalog of pointlike sources with near-infrared magnitudes $J=10.47, H=9.97$, and $K_{s}=9.83$. Assuming that this star is a physical binary companion, I can place it on the

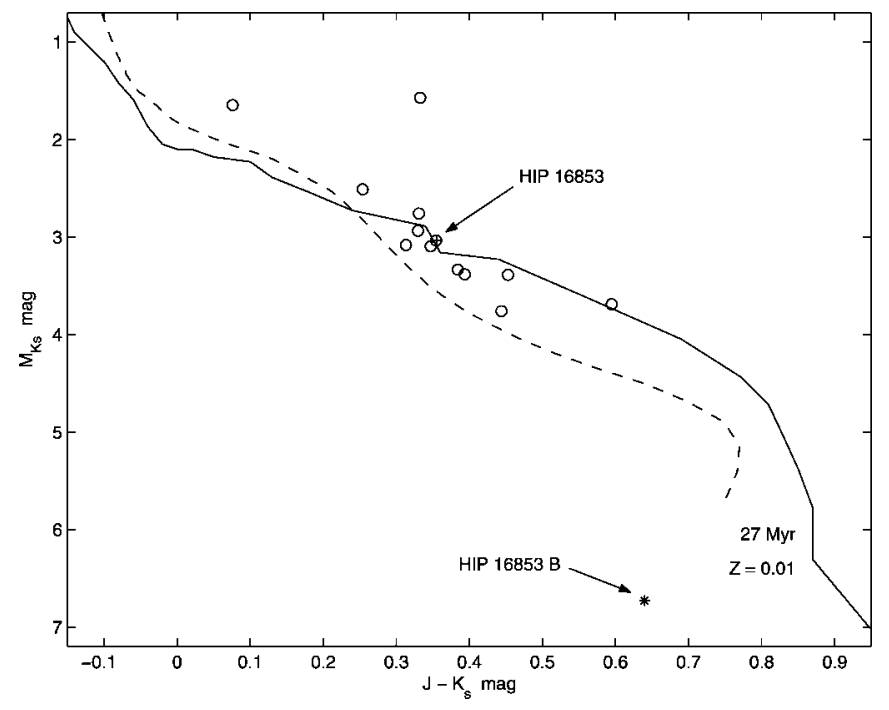

FIG. 1.-Near-infrared H-R diagram for bona fide members of the TucanaHorologium stream of young stars. Model isochrones from (Siess et al. 2000; solid line) and (Pietrinferni et al. 2004; dashed line) are drawn for age $27 \mathrm{Myr}, Z=0.01$, and no reddening. The position of the star HIP 16853 is indicated with a cross and a legend; the alleged wide companion HIP 16853 $\mathrm{B}$ is shown with an asterisk and a legend.

$\mathrm{H}-\mathrm{R}$ diagram in Figure 1, where its locus is indicated with an asterisk. Obviously, this result is inconsistent with the assumption of physical association with HIP 16853 and with the TUCHOR group. Furthermore, a proper motion for the B companion specified in the CCDM (of unknown origin) is completely inconsistent with the proper motion of the primary. Therefore, this companion is probably optical, and the closer astrometric companion HIP $16853 \mathrm{C}$ remains the only physical companion in this binary system.

The research described in this Letter was carried out at the Jet Propulsion Laboratory, California Institute of Technology, under a contract with the National Aeronautics and Space Administration. This research has made use of the SIMBAD database, operated at CDS, Strasbourg, France; and data products from the 2MASS, which is a joint project of the University of Massachusetts and the Infrared Processing and Analysis Center, California Technology Institute, funded by NASA and the NSF.

\section{REFERENCES}

Dommanget, J., \& Nys, O. 1994, Commun. Obs. R. Belgique, 115, 1

ESA. 1997, The Hipparcos and Tycho Catalogs, Vol. 1-17 (ESA SP-1200; Noordwijk: ESA)

Goldin, A., \& Makarov, V. V. 2006, ApJS, 166, 341

Henry, T. J., et al. 1996, AJ, 111, 439

Jancart, S., et al. 2005, A\&A, 442, 365

Makarov, V. V. 2007, ApJS, in press

Makarov, V. V., \& Kaplan, G. H. 2005, AJ, 129, 2420
Nordström, B., et al. 2004, A\&A, 418, 989

Pietrinferni, A., Cassisi, S., Salaris, M., \& Castelli, F. 2004, ApJ, 612, 168

Siess, L., Dufour, E., \& Forestini, M. 2000, A\&A, 358, 593

Torres, C. A. O., da Silva, L., Quast, G. R., de la Reza, R., \& Jilinski, E. 2000, AJ, 120, 1410

Torres, G., Guenther, E. W., Marschall, L. A., Neuhäuser, R., Latham, D. W., \& Stefanik, R. P. 2003, AJ, 125, 825

Zuckerman, B., \& Song, I. 2004, ARA\&A, 42, 685

Zuckerman, B., \& Webb, R. A. 2000, ApJ, 535, 959 